\title{
Germinação de sementes de Bothriospora corymbosa (Rubiaceae) recuperadas do trato digestório de Triportheus angulatus (sardinha) no Lago Camaleão, Amazônia Central
}

\author{
Lúcia Alencar MAIA ${ }^{1}$, Leocinira M. dos SANTOS $^{2}$, Pia PAROLIN ${ }^{3}$
}

RESUMO

$\mathrm{Na}$ Amazônia as áreas inundáveis são cobertas por florestas com alta diversidade de espécies arbóreas que proporcionam a ictiofauna frutos e sementes indispensáveis a sua alimentação. Alguns estudos de alimentação têm mostrado que a estrutura tegumentar de sementes encontradas no trato digestório de peixes, se apresentavam intactas. Assim, este estudo teve como objetivo verificar se Triportheus angulatus (Spix \& Agassiz, 1829) pode ser um agente dispersor de Bothriospora corymbosa (Bth) Hook. f. (Rubiaceae). A pesquisa foi desenvolvida no lago Camaleão, Ilha da Marchantaria, Manaus. Após a captura os peixes foram levados ao laboratório de campo, para a remoção do conteúdo do trato digestório, de onde foram retiradas 200 sementes para o experimento. As sementes do controle foram retiradas de frutos maduros. A germinação e emissão da radícula foram controladas diariamente. Neste estudo verificou-se que a principal fonte de alimento de T. angulatus foram frutos e sementes de espécies da várzea. $\mathrm{O}$ desempenho germinativo das sementes de $B$. corymbosa após a passagem pelo trato digestório de $T$. angulatus foi de $88,5 \%$ e do controle $95 \%$. Os Índices de Velocidade de Germinação (IVG) das sementes do trato digestório e do controle apresentaram diferenças significativas, porém, a passagem destas pelo intestino de T. angulatus não alterou a sua viabilidade. Assim, T. angulatus é um dos agentes dispersores de B. corymbosa, contribuindo com a distribuição desta espécie nas florestas de várzea da Amazônia Central.

\section{PALAVRAS-CHAVE}

Ictiocoria, Germinação de sementes, Triportheus angulatus, Bothriospora corymbosa, Floresta inundada da Amazônia.

\section{Seed germination of Bothriospora corymbosa (Rubiaceae) recouped of digestive tract of Triportheus angulatus (sardine) in Camaleão Lake, Central Amazonian}

\begin{abstract}
The Amazon floodplains are covered by forests with a high diversity of tree species that provides ictyofauna indispensable fruits and seeds for its feeding. Some studies of feeding have shown that the tegument structure of seeds found in the digestive tract of fish presented unbroken. Thus, this study had as objective to verify if Triportheus angulatus (Spix \& Agassiz, 1829) can be dispersal agent of Bothriospora corymbosa (Bth) Hook. f. (Rubiaceae). The research was developed in the Camaleão Lake, Island of the Marchantaria, Manaus. After the capture the fishes had been taken to the field laboratory, for the removal of the digestive tracts content, where 200 seeds for the experiment had been removed. The seeds of the control had been removed of ripe fruits. The germination and initial radicle emission were controlled daily. In this study it was verified that the main food source of T. angulatus had been fruits and seeds. The germinative performance of the seeds removed from digestive tract was of $88.5 \%$ and control $95 \%$. The Indices of Speed Germination (IVG) of the seeds which had passed through the digestive system and the control, presented significant differences, however, the passage of these through the intestine of T. angulatus did not modify its viability. Thus, T. angulatus is one of the dispersal agents of $B$. corymbosa, contributing with the distribution of this species in the floodplain forests of the Central Amazonian.
\end{abstract}

KEYWORDS

Ichthyochory, Seed germination, Triportheus angulatus, Bothriospora corymbosa, Amazon floodplain forest.

IInstituto Nacional de Pesquisas da Amazônia (INPA), CPBO. Caixa Postal 478. Manaus, AM. Brasil; alencar@inpa.gov.br. ${ }^{2}$ Bolsista CNPq/INPA-PIBIC.

${ }^{3}$ Max-Planck-Institute for Limnology, Tropical Ecology, Plön, Alemanha; pparolin@mpil-ploen.mpg.de 


\section{INTRODUÇÃO}

A região Amazônica é caracterizada, principalmente, pela sua vasta bacia hidrográfica e pela floresta tropical úmida (Pires, 1974; Pires \& Prance, 1985). As áreas inundáveis da Amazônia estão associadas aos grandes rios (Amazonas, Solimôes, Negro e Tapajós) e correspondem a um total de 6\% da área da Amazônia brasileira, num total aproximado de $300.000 \mathrm{Km}^{2}$, sendo cerca de $200.000 \mathrm{Km}^{2}$ de várzeas e $100.000 \mathrm{Km}^{2}$ de igapós (Junk, 1993).

A várzea é um dos ecossistemas mais ricos da Amazônia em produtividade biológica, biodiversidade e recursos naturais. Alguns estudos estimam cerca de 1.500 espécies de peixes identificadas e outros acreditam que existam entre três a quatro mil espécies de peixes na bacia Amazônica (Junk et al., 1983; Goulding, 1993; Salati et al., 1998). O recurso pesqueiro representa a síntese das interaçōes entre os diversos componentes desse ecossistema, além de ser à base da dieta e principal fonte de renda da população ribeirinha (Furtado, 1988; Petrere, 1992; Batista et al., 2000; Maia, 2001). Vários estudos comprovam a intensa interação da floresta alagável e a comunidade de peixes na Amazônia (Gottsberger, 1978; Goulding, 1980; Leite, 1987; Maia, 1997; Waldhoff \& Maia, 2000; Santos et al., 2004).

Nestas áreas os vegetais apresentam várias adaptações, entre estas, as relacionadas à produção frutos e dispersão de sementes (Kubitzki \& Ziburski, 1994; Maia, 1997; Maia \& Jackson, 2000; Parolin, 2001; Parolin et al., 2004; Maia et al.,_2005). O período de produção pode variar nas comunidades florísticas e está relacionada às mudanças das condiçōes ambientais e as fenofases reprodutivas (Ziburski \& Kubitzki, 1994; Maia, 1997; Wittmann \& Parolin, 1999; Santos et al., 2003a). Muitas árvores frutificam durante o período de alagação (Maia, 1997; Maia \& Chalco, 2002; Schöngart et al. 2002) e alguns frutos ao caírem na água permanecem flutuando, favorecendo a dispersão das sementes por hidrocoria e ou ictiocoria (Kubitzki \& Ziburski, 1994; Maia, 1997). Entre os mecanismos de dispersão de sementes encontram-se os peixes que atuam como condutores de diásporos (Gottsberger, 1978; Goulding, 1980; Santos \& Maia, 2004) favorecendo uma alta diversidade de espécies nas florestas alagáveis (Janzen, 1976).

O consumo de biomassa vegetal pelos peixes é alto durante o período de cheia (Pizango-Paima, 2001; Maia \& Chalco, 2002) ao entrarem nas áreas alagadas se alimentam de detritos vegetais, folhas jovens, frutos, sementes, etc., que caem na água, demonstrando ser a bioprodutividade e em especial a produção de frutos, item importante na cadeia alimentar (Maia, 1997; Silva \& Maia, 1999; Waldhoff \& Maia, 2000).

Em relação ao consumo de sementes, foi observado que a estrutura tegumentar de algumas sementes encontradas no trato digestório dos peixes apresentavam-se intactas (Goulding, 1980; Pilati, et al., 1999; Santos \& Maia, 2004; Santos et al., 2003b). Entretanto, pouco se conhece em relação às taxas de germinação, IVG e viabilidade de sementes após a passagem pelo trato digestório de peixes, como também, aqueles que são os dispersores ou predadores de sementes.

O aumento demográfico e a agricultura têm se intensificado e em algumas áreas é visível à degradação do ecossistema nas margens dos rios, causando alterações a médio e longo prazo na diminuição da oferta de alimentos para a fauna aquática. Neste contexto, o estudo da germinação e dispersão de espécies típicas da floresta de várzea, como Bothriospora corymbosa (Bth.), se reveste de particular importância para melhorar o entendimento dos processos da dinâmica de populaçôes, do manejo sustentável e recuperação das áreas alagáveis degradadas da Amazônia Central.

Assim, este estudo apresenta resultados referentes a identificação dos conteúdos do trato digestório de Triportheus angulatus; a determinação do potencial germinativo e Índice de Velocidade de Germinação (IVG) das sementes de $B$. corymbosa após a passagem pelo trato digestório deste peixe e a atuação de $T$. angulatus como dispersor de $B$. corymbosa em áreas alagáveis da Amazônia Central.

\section{MATERIAL E MÉTODOS}

\section{ÁREA DE COLETA}

As sementes de Bothriospora corymbosa e os exemplares de Triportheus angulatus foram obtidos no lago Camaleão, Ilha da Marchantaria, Município de Iranduba-AM, entre $03^{\circ} 15^{\prime}$ S e $59^{\circ} 58^{\prime}$ W. Parte da ilha é coberta por vegetação de floresta de várzea, com formações arbóreas, arbustivas e herbáceas (macrófitas aquáticas e semi-aquáticas). A flutuação anual média do nível da água é cerca de $10 \mathrm{~m}$ (Junk, 1989), a fase aquática iniciou em dezembro, atingindo a cota máxima em julho; a vazante começou em agosto e a fase terrestre deu-se entre setembro e dezembro.

\section{DESEMPENHO GERMINATIVO DAS SEMENTES}

Os exemplares de T. angulatus foram capturados em agosto e setembro de 2003 (vazante), utilizando-se malhadeiras de vários tamanhos, variando entre 30 e $70 \mathrm{~mm}$. Após a captura dos 30 exemplares de T. angulatus, com tamanho padrão entre 07 e $19 \mathrm{~cm}$ de comprimento, foram levados ao laboratório de campo, e através de inserção ventral foram retirados os tratos digestórios para a análise dos conteúdos estomacais $(n=30)$ e verificação da dieta alimentar. Para a determinação do potencial germinativo e Índice de Velocidade de Germinação (IVG) foram retiradas 200 sementes, ao acaso, do trato digestório (intestino), lavadas em água corrente, contadas e acondicionadas em placas de Petri, revestidas 
com papel especial para germinação CEL-065 (distribuídas de maneira uniforme). Cada tratamento foi constituído de quatro repetiçôes, com 50 sementes cada, armazenadas ao ar livre no Campus I do INPA-Manaus, numa área de vegetação secundária, sujeita a precipitação, com temperatura, luz e umidade relativa ambiente (Figura 1). A umidade foi mantida diariamente com água destilada.

O desempenho germinativo das sementes foi calculado através da relação: $P G=(S G * 100) / A M$, sendo $P G=$ Porcentagem de Germinação; SG = Número de Sementes Germinadas; $\mathrm{AM}=$ Total de Sementes da Amostra. As freqüências germinativas foram contadas em intervalos consecutivos de 24 horas, até que a taxa diária de germinação permanecesse em zero por pelo menos uma semana. As sementes foram consideradas germinadas após emissão da radícula e a contagem foi efetuada diariamente durante 07 dias, até estabilização. Para o controle, foram coletados frutos maduros de B. corymbosa (de árvores matrizes) e retiradas as sementes ( $\mathrm{n}=200)$. Os tratamentos de verificação do potencial germinativo e IVG seguiram a mesma metodologia utilizada para as sementes recuperadas do trato digestório (intestino). $\mathrm{O}$ Teste de Tukey foi realizado para verificar se existia diferença estatística entre os tratamentos ao nível de 5\% de probabilidade.

O material fértil foi coletado e depositado no Herbário do INPA, nº 215.120. A identificação botânica foi efetuada através de comparação com exsicatas depositadas no Herbário do INPA e consulta a especialistas.

\section{RESULTADOS E DISCUSSÃO}

\section{DIETA ALIMENTAR DE Triportheus angulatus}

A espécie apresentou espectro alimentar diversificado, constituído de frutos, sementes, fragmentos vegetais e insetos. Os principais frutos e sementes consumidos foram: sardinheira (Bothriospora corymbosa), tarumã (Vitex cymosa Bertero ex Spreng.), imbaúba (Cecropia latiloba Miq.), e munguba (Pseudobombax munguba Mart. et Zucc.) Dugand; fragmentos vegetais: pedaços de folhas e flores, frutos, sementes e raízes; insetos adultos: hymenopteros (formigas), pedaços de coleópteros, dípteros e hemípteros.

Triportheus angulatus apresenta sazonalidade em sua dieta, durante a enchente é expressivo o consumo de insetos e restos vegetais, que são disponibilizados com a inundação das áreas colonizadas pela vegetação herbácea, semi-terrestre e terrestre (Yamamoto, 2001). A dieta alimentar de muitas espécies de peixes está relacionada com a dinâmica do pulso de inundação, uma vez que este influencia o ciclo fenológico reprodutivo de espécies vegetais e conseqüentemente a oferta de alimento (Maia, 1997; 2001). Na enchente, especialmente durante as águas altas, a oferta de alimento favoreceu a intensa

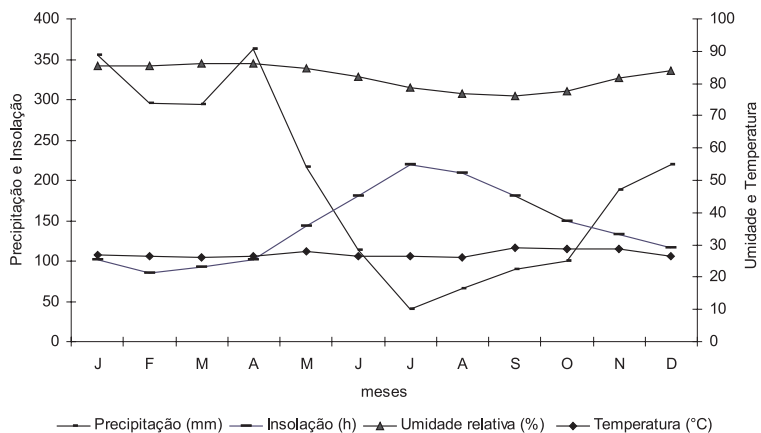

Figura 1 - Média da soma mensal de temperatura $\left({ }^{\circ} \mathrm{C}\right)$, umidade relativa (\%), insolação (h), precipitação (mm) em 2003. Os dados de temperatura, umidade relativa, insolação e precipitação foram obtidos no $1^{\circ} \mathrm{DISME} / \mathrm{AM} /$ AC/RR-Manaus-AM.

atividade alimentar de $T$. angulatus no lago em estudo. Segundo Yamamoto (2001) e Santos et al. (2003b) este peixe consumiu preferencialmente, no lago Camaleão, insetos na seca e frutos e sementes na cheia e vazante, constituindo-se um explorador efetivo da floresta.

\section{TESTES DE GERMINAÇÃO}

As sementes retiradas do trato digestório apresentaram $88,5 \%$ de germinação (Tabela 1). O início e pico de germinação ocorreram no $02^{\circ}$ dia da semeadura, com 147 sementes germinadas e o final da germinação deu-se no $19^{\circ}$ dia. Após o segundo dia os valores decresceram (Figura 2). O desempenho germinativo das sementes de B. corymbosa do controle foi de $95 \%$ (Tabela 1). O início e pico de germinação ocorreram aos $04^{\circ}$ e $06^{\circ}$ dias, respectivamente, e a curva apresentou maior decréscimo a partir do $07^{\circ}$ dia (Figura 2). Em termos absolutos o poder germinativo e emissão de radículas nas sementes mostraram-se superior no controle. Entretanto, o Teste Tukey mostrou que não há diferença significativa a $5 \%$ de probabilidade, entre o desempenho

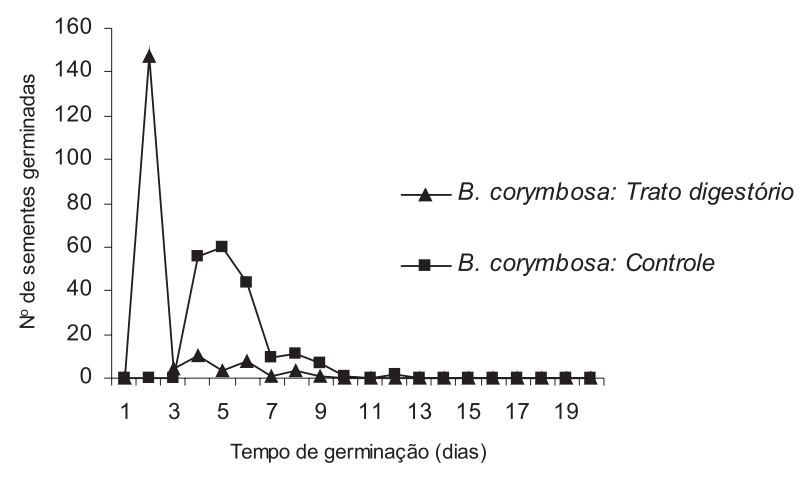

Figura 2 - Curvas de germinação de sementes de $B$. corymbosa recuperadas do trato digestório de $T$. angulatus $(n=200)$ e controle $(n=200)$ em relação ao tempo de germinação (dias). 
germinativo das sementes de $B$. corymbosa recuperadas no trato digestório (intestino) de T. angulatus e daquelas obtidas diretamente dos frutos.

Tabela 1 - Características da germinação de sementes Bothriospora corymbosa passadas pelo trato digestório de Triportheus angulatus e do controle (sem tratamento). Características avaliadas: germinação (\%) e IVG.

\begin{tabular}{lll}
\hline \multirow{2}{*}{ Tratamentos } & \multicolumn{2}{c}{ Características avaliadas } \\
\cline { 2 - 3 } & Germinação (\%) & $\begin{array}{l}\text { Índice de velocidade } \\
\text { de germinação (IVG) }\end{array}$ \\
\hline Controle & $95,0 \mathrm{a}$ & $9,26 \mathrm{~b}$ \\
Trato digestório de sardinha & $88,5 \mathrm{a}$ & $19,98 \mathrm{a}$ \\
\hline Desvio Padrão & 5.24 & 0.05 \\
C.V (\%) & 7.00 & 1.24 \\
DMS (Tukey) & 9.07 & 0.08 \\
\hline
\end{tabular}

*Médias seguidas da mesma letra na coluna não diferem a $5 \%$ de probabilidade pelo teste de Tukey.

Alguns estudos mostraram que sementes recuperadas do trato digestório de peixes estavam aptas a germinar. Goulding (1980) verificou a viabilidade de sementes retiradas do intestino de Mylossoma (pacu); Bevilacqua et al., (1995) encontraram 65\% de germinação em Cecropia sp. após serem ingeridas por Auchenipterichthys longimanus (peixe gato); Pilati et al. (1999) verificaram que sementes de C. pachystachya retiradas do trato digestório de Ptedoras granulosus, apresentaram valores de germinação de $90,35 \%$ na porção média do intestino, $89,25 \%$ na porção posterior e $95,38 \%$ na anterior e Santos et al. (2003b) obtiveram germinação em $88,7 \%$ das sementes de C. latiloba recuperadas do trato digestório de Brycon cephalus (matrinxã). Neste estudo, o desempenho germinativo das sementes de $B$. corymbosa recuperadas do trato disgetório $(88,5 \%)$ mostrou que a passagem destas pelo intestino de $T$. angulatus não alterou a viabilidade das sementes, estando de acordo com os resultados encontrados pelos autores acima citados.

O fruto de B. corymbosa é uma baga globosa de 0,6-0,8 $\mathrm{cm}$, polpa carnosa, as semente são pequenas $(0,15 \times 0,20 \mathrm{~cm})$ com envoltório carnoso e tegumento resistente. $\mathrm{O}$ tegumento pode ter influenciado na maior capacidade de suportar a destruição por maceração, trituração e ou pela ação dos ácidos digestórios de T. angulatus. Estes fatos impediram a destruição das sementes e mantiveram as altas porcentagens de germinação. Segundo Goulding (1980) sementes pequenas ingeridas por peixes estavam envolvidas por material carnoso e sem digestão.

Em relação ao IVG os resultados mostraram que há diferenças significativas a $5 \%$ de probabilidade pelo teste de Tukey, entre o IVG das sementes de B. corymbosa recuperadas do trato digestório de $T$. angulatus (IVG $=19,98)$ e daquelas obtidas diretamente dos frutos (IVG $=9,26)$, (Tabela $1 \mathrm{e}$ Figura 3). A ação dos ácidos no sistema digestório do peixe podem ter favorecido para o aumento do IVG. Entretanto, estas diferenças não influenciaram no desempenho germinativo das sementes.

\section{DISPERSÃO DE SEMENTES}

Os processos de migração trófica para a busca de recursos alimentares e de migração reprodutiva da ictiofauna no lago Camaleão estão correlacionados às flutuaçôes sazonais e à disposição dos recursos alimentares (Yamamoto, 2001). Gottsberger (1978) menciona que a variação sazonal do nível d'água pode ser um importante parâmetro ecológico de dispersão hidrocórica, embora a maioria das espécies produza frutos que têm características especiais para a dispersão animal, como o desenvolvimento de mesocarpo comestível ou arilo. Goulding (1980) examinou 40 espécies de frutos da várzea da bacia do rio Madeira e verificou que 35 destes tinham capacidade de flutuação, pelo menos por algum tempo, e chama atenção para o fato de que a flutuação pode ser o mecanismo mais importante para a dispersão de sementes nas florestas inundadas da Amazônia. A flutuação aumenta a distancia percorrida pelas sementes e favorece a probabilidade de serem ingeridas por peixes (Gottsberger, 1978; Santos et al., 2003b).

Nesse sentido, as estratégias reprodutivas de $B$. corymbosa, produção de frutos na fase aquática, tem em $T$. angulatus um efetivo dispersor, uma vez que o processo de migração trófica deste peixe, no lago Camaleão, coincide com a fenofase de frutificação deste vegetal.

A propagação de sementes através do ambiente pode afetar os padrões de estabelecimento de plântulas e a composição

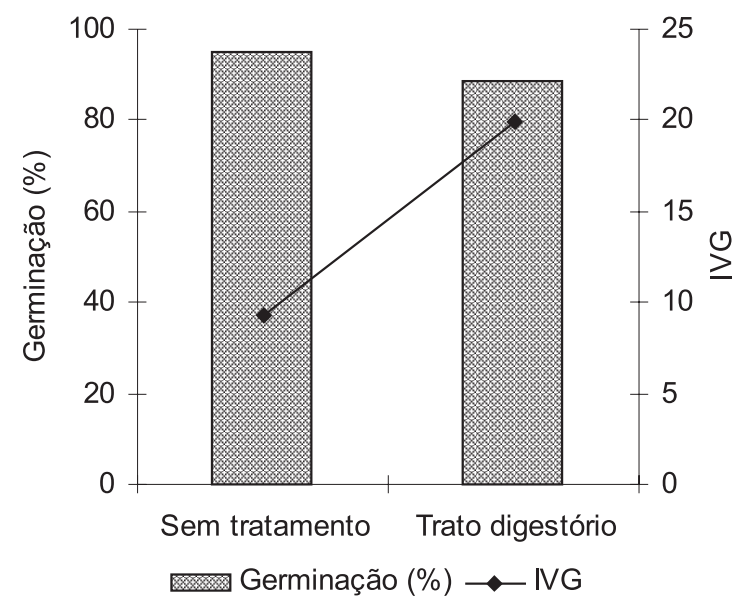

Figura 3 - Comportamento germinativo das sementes $B$. corymbosa passadas pelo trato digestório de $T$. angulatus e daquelas obtidas diretamente dos frutos (sem tratamento). Características avaliadas: Germinação (\%) e Índice de Velocidade de Germinação (IVG). 
florística da população, tendo importantes conseqüências demográficas na distribuição e abundância de espécies. Segundo Howe \& Smallwood (1982) a dispersão de sementes aumenta a probabilidade de estabelecimento de indivíduos em condiçôes ambientais favoráveis, já que algumas delas poderiam cair em áreas onde a germinação, o crescimento e a sobrevivência de plântulas são propícias.

A dispersão e a predação de sementes são fatores importantes para a manutenção da espécie (Jansen, 1976). A dispersão dá início ao processo de sucessão vegetal e dependendo do nível de predação, o sucesso da recuperação de uma área degradada poderá ser assegurado. Gottsberger (1978), Goulding (1980), Pilati et al. (1999) e Santos \& Maia (2004) observaram que muitos vegetais nas áreas inundáveis têm os peixes como dispersores potenciais ou efetivos de suas sementes.

\section{CONCLUSÕES}

Verificou-se que não houve diferença significativa entre o desempenho germinativo das sementes de Bothriospora corymbosa recuperadas no trato digestório (intestino) de Triportheus angulatus e aquelas obtidas diretamente dos frutos.

O Índice de Velocidade de Germinação (IVG) das sementes de $B$. corymbosa recuperadas no trato digestório (intestino) de T. angulatus é superior daquelas retiradas dos frutos.

A alta viabilidade das sementes recuperadas no trato digestório de T. angulatus e o fato deste peixe realizar migrações, caracteriza o mesmo como dispersor de sementes de B. corymbosa, podendo contribuir para ampliar a área de colonização ou de ocupação desta espécie.

\section{BIBLIOGRAFIA CITADA}

Batista, V.S.; Freitas, C.E.C.; Silva, A.J.I.; Brasil, D.F. 2000. The fishing activity of the river people in the floodplain of the Central Amazon. In: Junk, W.J.; Ohly,J.J.; Piedade, M.T.F.; Soares, M.G. (Eds.): The Central Amazon Floodplain: Actual Use and Options for a Sustainable Management. Backhuys Publishers, Leiden. pp. 417-431.

Bevilacqua, G.D.; Caramaschi, E.P.; Scarano, F.R. 1995. Germinabilidade de sementes de Cecropia sp., coletadas no trato digestório de Auchenipterichthys longimanus (Siluriformes Auchenipteridae). In: XI Encontro Brasileiro de Ictiologia. Resumos. PUC de Campinas, Campinas, SP. p 13.

Furtado, 1988. Os caboclos pescadores do baixo rio Amazonas e o processo de mudança social e econômica. In: Diegues, A.C (ed.). Ciências Sociais e o Mar do Brasil. II PPCAUB, São Paulo, SP. p. 180- 203.

Gottsberger, G. 1978. Seed dispersal by fish in the inundated regions of Humaitá, Amazonia. Biotropica, 10(3):170-183.
Goulding, M. 1980. The fishes and the forest. Explorations in Amazonian natural history. Univ. of California Press.Berkeley, CA, USA. 280pp.

Goulding, M. 1993. Flood forets of the Amazon. Scientific American, 268(3):114-120.

Howe, H.F.; Smallwood J. 1982. Ecology of seed dispersal. Annu. Rev. Ecol. Syst., 13:201-228.

Janzen, D.H. 1976. Seeding patterns of tropical trees. In: Tomlinson, P.B.; Zimmermann, M.H. (Eds.). Tropical trees as living systems. Cambridge University Press. Cambridge. p. 83-128.

Junk, M.J.; Soares, G.M.; Carvalho, F.M. 1983. Distribution of fish species in a lake of the Amazon river flooplain near Manaus (lago Camaleão) with special reference to extreme oxygen conditions. Amazoniana, 7(4): 397-431.

Junk, W.J. 1989. Flood tolerance and tree distribution in Central Amazonian floodplains. In: Nielsen, L.B.; Nielsen, I.C.; Balslev, H. (Eds.). Tropical forests: Botanical dynamics, speciation and diversity. Academic Press, London. p. 47-64.

Junk, W.J. 1993. Wetlands of tropical South America. In: Whigham D.F. et al. (Eds.). Wetlands of the World I. Kluwer Academic Publ. Netherlands. p. 679-739.

Kubitzki, K.; Ziburski A. 1994. Seed dispersal in flood plain forests of Amazonia. Biotropica, 26(1): 30-43.

Maia, L.M.A. 1997. Influência do pulso de inundação na fisiologia, fenologia e produção de frutos de Hevea spruceana (Euphorbiaceae) $e$ Eschweilera tenuifolia (Lecythidaceae) em área inundável de igapó da Amazônia Central. Tese de doutorado. Instituto Nacional de Pesquisas da Amazônia/Universidade Federal do Amazonas, Manaus, AM. 186pp.

Maia, L.M.A.; Jackson, M.B. 2000. Morphological and growth responses of woody plant seedlings to flooding of the Central Amazon floodplain forest. Verb. Internat. Verein. Limnol., 27(4):1711-1716.

Maia, L.M.A. ; Chalco F.P. 2002. Produção de frutos de espécies da floresta de várzea da Amazônia central importantes na alimentação de peixes. Acta Amazônica, 32(1): 45-54.

Maia, L.M.A. 2001. Frutos da Amazônia: Fonte de alimento para peixes. Editora SEBRAE. Manaus, AM. 143pp.

Maia, L.M.A; Maia, S; Parolin, P. 2005. Seedling Morphology of non-pioneer trees in central Amazonian várzea floodplain Forest. Ecotropica, 11:1-8.

Parolin, P. 2002. Fugitive and possessive establishment strategies in Amazonian floodplain pioneers. Flora, 198: 436-443.

Parolin, P. 2001. Morphological and physiological adjustments to waterlogging and drought in seedlings of Amazonian floodplain trees. Oecologia, 128: 326-335.

Parolin, P. ; Ferreira, L.V. ; Junk, W.J. 2003. Germination characteristics and establishment of trees from Central Amazonian floodplains. Tropical Eccology, 44(22): 155-167.

Parolin, P. ; De Simone O. ; Haase K. ; Waldhoff D. ; Rottenberger S. ; Kuhn U. ; Kesselmeier J. ; Schmidt W. ; Piedade M.T.F. ; Junk W.J. 2004. Central Amazon floodplain forests: tree survival in a pulsing system. The Botanical Review, 70(3): 357-380. 
Pilati, R.; Adrian I.D.; Carneiro J.W.P. 1999. Performance of seed germination of Cecropia pachystachya Trec. (Cecropiaceae) from the digestive tract of Ptedoras granulosus (Valenciennes, 1833) of the floodplain of the Upper Paraná River. Interciencia, 24(6): 381-388.

Pires, J.M. 1974. Tipos de vegetação da Amazônia, Belém. Brasil Florestal, 5: 179-202.

Pires, J.M.; Prance G.T. 1985. The vegetation types of the Brazilian Amazon. In: Prance G.T.; Lovejoy T.E. (Eds.) Amazonia Key environments. Pergamon Press. London. pp. 109-145.

Pizango-Paima, E.G. 2001. Composição corporal e alimentar do matrinxã, Brycon cephalus (Gunther, 1869) na Amazônia Central. Acta Amazonica, 31(3): 509-520. 2001.

Petrere, Jr. M. 1992. As comunidades humanas ribeirinhas da Amazônia e suas transformaçôes sociais. In: Diegues, A.C (Ed.). Ciências Sociais e o Mar do Brasil. II PPCAUB, São Paulo, SP. p. 31-68.

Salati, E.; Santos, A. A ; Lovejoy, T. E. Klabin, I. 1998. Porque Salvar a Floresta Amazônica? INPA - Manaus, AM. 114 pp.

Santos, L.M; Maia, L.M.A ; Fernandez, M. C. 2003a. Fenologia de Bothriospora corymbosa (Rubiaceae) em floresta de várzea da Amazônia Central. In: $4^{o}$ Encontro de Genética do Amazonas. Primeiro Encontro de Genética da Regiāo Norte. Manaus-AM. $1 \mathrm{p}$.

Santos, L.M.; Maia, L.M.A.; Soares, M.G. 2003b. Germinação de sementes de Bothriospora corymbosa recuperadas do trato digestório de Triportheus angulatus (sardinha) da Amazônia Central. In: XII Jornada de Iniciação Científica, PIBIC/INPA. pp 39-40.

Santos, L. M.; Maia, L.M.A. 2004. Germinação de sementes de Vitex cymosa Bertero ex Spreng. recuperadas do trato digestório de Triportheus angulatus (SPIX, 1829) sardinha no lago Camaleão - Amazônia Central. In: XIII Jornada de Iniciação Científica, PIBIC/INPA. pp 39-40.
Santos, L.M.; Maia, L.M.A.; Chalco, M. F. 2004. Germinação de sementes de Cecropia latiloba Miq. recuperadas do trato digestório de Brycon cephalus (matrinxã) no lago CamaleãoAmazônia Central. In: $3^{\circ}$ Simpósio Brasileiro de Pós-Graduação em Engenharia Florestal, $1^{\circ}$ Encontro Amazônico de Ciências Florestais. Manaus - AM. 01pp.

Schöngart, J., Piedade M.F.T., Ludwigshausen S., Horna V.; Worbes M. 2002. Phenology and stem-growth periodicity of tree species in Amazonian floodplain forests. Journal of Tropical Ecology, 18: 581-597.

Silva, O.M.A.; Maia, L.M.A. 1999. Identificação botânica de seis espécies importantes na alimentação de peixes comerciais da Amazônia Central. In: VIII Jornada de Iniciação Científica, PIBIC/INPA, p 27-29.

Waldhoff, D.; Maia L.M.A. 2000. Production and chemical composition of fruits from trees in floodplain forests of Central Amazonia and their importance for fish production. In: Junk, W.J.; Ohly, J.J.; Piedade, M.T.F.; Soares, M.G. (Eds.): The Central Amazon Floodplain: Actual Use and Options for a Sustainable Management. Backhuys Publishers, Leiden, p. 393-415.

Wittmann, F.; Parolin, P. 1999. Phenology of six tree species from Central Amazonian

várzea. Ecotropica, 5:51-57.

Yamamoto, K. C. 2001. Regime alimentar de Triportheus angulatus (Spix \& Agassiz, 1829) e a concentração de oxigênio em lago de várzea da Amazônia Central, AM-Brasil. Monografi a Curso de Engenharia de Pesca. Universidade do Amazonas. Manaus, AM. 27pp.

Ziburski, A.; Kubitzki, K. 1994. Seed Dispersal in Flood Plain Forest of Amazônia. Biotropica, 26 (1):30-43.

Recebido em 02/08/2005

Aceito em 29/06/2007 